\title{
EDUCATION
}

Research, Innovation and Solutions on-line ${ }^{\circledR}$

\section{Evaluación del aprendizaje en la universidad: una mirada retrospectiva y prospectiva desde la divulgación científica}

\author{
Ibis Álvarez Valdivia \\ Departamento de Psicología Evolutiva y de la Educación, \\ Universidad Autónoma de Barcelona
}

\section{España}

Dra.Ibis Álvarez Valdivia. Universidad Autónoma de Barcelona, 08193. Bellaterra (Cerdanyola del Vallés).

España. E-mail: ibismarlene.alvarez@uab.es

(C) Education y Psychology $\mathrm{I}+\mathrm{D}+\mathrm{i}$ and Editorial EOS (Spain) 


\section{Resumen}

Este artículo tiene como propósito aproximarse al estado actual de la investigación sobre la evaluación del aprendizaje en la universidad. Se realiza una búsqueda en publicaciones científicas periódicas de reconocido prestigio. Tres preguntas se elaboran a modo de guía del análisis de contenido de los artículos consultados: (i) ¿Cuáles son las problemáticas relacionadas con la evaluación del aprendizaje en la universidad que vienen preocupando a docentes e investigadores en la última década?, (ii) ¿Cómo se manifiestan en diferentes contextos universitarios? y (iii) ¿Qué sugieren las investigaciones como soluciones pertinentes y probables para mejorar la evaluación del aprendizaje en la universidad? La categorización del contenido de los artículos gira en torno a ello. Con esta información se crea la base documental para este trabajo que permite realizar algunas inferencias, presumiblemente de interés, respecto a cuáles son las problemáticas relacionadas con la evaluación del aprendizaje en la universidad que vienen preocupando a docentes e investigadores en la última década; cómo se manifiestan en diferentes contextos universitarios y qué sugieren las investigaciones como soluciones pertinentes y probables. Finalmente se ofrece una amplia lista de referencias bibliográficas, útil a docentes e investigadores interesados en el tema.

Palabras Clave: Evaluación, Aprendizaje, Educación Superior. 


\begin{abstract}
This article aims to give insight into the present situation of research in learning assessment in Higher Education. It is based on a study done by skimming through articles issued in prestigious periodical scientific publications. As a guide for the analysis of the content of the reviewed articles, three questions were taken into consideration: (i) What are the main problems related to learning assessment in the university that teachers and researchers are concerned about in the last decade?, (ii) How do they manifest in different university contexts? and (iii) What are the possible and pertinent solutions to improve learning assessment? The content analysis was based on the answers to these main questions. Information obtained from this analysis allowed us to make some inferences, perhaps of great interest, in respect to the problems that teachers and researchers are concerned about learning assessment in the last decade, how these problems are manifested in higher education institutions and the possible solutions suggested in the research works on the topic. Finally, an extensive, useful list of references is offered to all the teachers and researchers interested in the topic
\end{abstract}

Key words: Assessment, Learning, Higher Education,

Received: 01/09/08 Initial acceptance: 02/19/08 Final acceptance: 03/30/08 


\section{Introducción}

La evaluación del aprendizaje es una actividad compleja que forma parte del proceso docente y siempre ha constituido una preocupación central. A pesar de su incuestionable función, sin temor a demasiado equívoco, podría afirmarse que tanto para los docentes como para los estudiantes, la evaluación se percibe como el componente más incómodo del proceso de enseñanza y aprendizaje, del cual, la mayoría de las veces, se querría prescindir. Sin embargo, es esencial contar con un sistema de evaluación que legitime y promueva la calidad de la enseñanza y el aprendizaje universitario. Por otra parte, no hay que olvidar que la repercusión de la evaluación es tal que, hoy en día, es considerada como un importante pilar de la enseñanza universitaria; diferentes estudios han comprobado que la evaluación determina el aprendizaje de los estudiantes y no el currículo oficial (Biggs, 2005).

A la luz de los cambios que ha traído la entrada en el Siglo XXI se ha hecho más patente en la investigación y en la innovación docente este sensible tópico en todos los ámbitos educativos. Al respecto, estudio realizado por Broabfoot y Black (2004) cuestiona los modos predominantes de evaluación en tanto tienden a reforzar nociones sobre la concepción del currículo y del aprendizaje no acordes con las demandas educativas actuales (aprender a aprender, aprendizaje basado en competencias, aprendizaje para la vida, etc.). Este estudio advierte que la investigación sobre la evaluación educativa ha estado enfocando en grado excesivo las técnicas, en lugar del análisis de su relación con la calidad del aprendizaje.

En el ámbito universitario esta problemática es de sensible interés en medio de las reformas que se promueven a favor de cambios en la concepción del currículo, del aprendizaje y consecuentemente de la evaluación (Billing, 2007; González, 2006; Jongbloed, 2002; Mollis y Marginson, 2002). Diversas investigaciones han puesto de manifiesto que la práctica de la evaluación es disfuncional y desequilibrada en muchos aspectos En general se evalúa estereotipadamente, la evaluación es incoherente con el proceso de enseñanza-aprendizaje o independiente del mismo, no retroalimenta estos procesos y no informa al alumnado de las condiciones de la evaluación (Brown y Glasner, 2003; González, 2003; Segers y Dochy, 1996). El estado actual de la cuestión sugiere la emergencia de un nuevo paradigma de evaluación basado en epistemologías diferentes y en las necesidades educativas de los estudiantes universitarios de la sociedad contemporánea. 
Considerando la actualidad y el interés de este tema, el estudio que se presenta en este artículo establece como propósito realizar una búsqueda de investigaciones que focalizan la evaluación del aprendizaje en la universidad, en publicaciones científicas de relevante impacto $\mathrm{y}$, sobre esta base, extraer algunas conclusiones relativas a las problemáticas y aportaciones que se han hecho notar en los últimos años cuando se aborda este tema.

Las preguntas que guiaron la indagación en este tema fueron las siguientes:

- ¿Cuáles son las problemáticas relacionadas con la evaluación del aprendizaje en la universidad que vienen preocupando a docentes e investigadores en la última década?

- ¿Cómo se manifiestan en diferentes contextos universitarios?

- ¿Qué sugieren las investigaciones como soluciones pertinentes y probables para mejorar la evaluación del aprendizaje en la universidad?

\section{Método}

\section{Diseño y procedimiento}

El criterio que guió la clasificación inicial de los artículos fue el enunciado que conforma su título. Realizado este agrupamiento las referencias de los artículos seleccionados se editan en un documento Word con hipervínculos, desde su título a la Web de la revista en cuestión. Este recurso facilita la consulta de los resúmenes de los artículos que es de libre acceso a través de la Web, este modo de proceder agiliza el análisis de su contenido. Por último se seleccionan fragmentos de textos de los resúmenes, suficientemente elocuentes, respecto a las problemáticas o sugerencias que presentan los artículos consultados. En algunos casos, por su interés, se consulta el artículo completo y otras fuentes documentales citadas en su corpus.

El resultado de este proceso permite la codificación de los artículos seleccionados y la creación de la base documental para su análisis. El propósito final es realizar algunas inferencias, presumiblemente de interés, respecto a cuáles son las problemáticas relacionadas con la evaluación del aprendizaje en la universidad que vienen preocupando a docentes e investiga- 
dores en la última década; cómo se manifiestan en diferentes contextos universitarios y qué sugieren las investigaciones como soluciones pertinentes y probables.

\section{Materiales}

Para conseguir el objetivo de este estudio la búsqueda de referencias científicas sobre la evaluación en la universidad se realiza en tres revistas: 1) Higher Education, 2) Assessment y Evaluation in Higher Education y 3) The Electronic Journal of Research in Educational Psychology (Revista Electrónica de Investigación Psicoeducativa, REIPE). Estas revistas se seleccionan por su prestigio entre profesionales e investigadores del mundo universitario. La revisión focaliza los artículos que abordan el tópico de la evaluación del aprendizaje y se limita a las publicaciones de la última década, a partir de 1996, período en el que se nota un auge importante de publicaciones al respecto. La tabla 1 muestra la cantidad de trabajos que para este estudio se consideraron de interés.

Tabla 1. Resumen del material consultado

\begin{tabular}{ccc}
\hline Fuente & $\begin{array}{c}\text { Volúmenes } \\
\text { consultados }\end{array}$ & $\begin{array}{c}\text { Artículos sobre evaluación del } \\
\text { aprendizaje en la universidad }\end{array}$ \\
Higher Education & $\begin{array}{c}\text { Del 33 al 53 } \\
(1996-2007)\end{array}$ & 24 \\
Education & Del 21 al 32 & 10 \\
Assessment y Evaluation in Higher & $(1996-2007)$ & \\
The Electronic Journal of Research & Del 1 al 5 & \\
in Educational Psychology & $(2003-2007)$ & \\
\hline
\end{tabular}

$\mathrm{N}=275$

'Higher Education' está reconocida como la principal revista internacional sobre estudios de Educación Superior. Desde su surgimiento en 1972 ha seguido el desarrollo educativo en el ámbito universitario de todo el mundo. Publica ocho números cada año, los artículos abordan problemáticas relativas a docentes y estudiantes así como de planificación y gestión en la Educación Superior. El tema de la evaluación del aprendizaje aparece como promedio dos veces por año, en su mayoría relativos a la coherencia de su concepción y práctica con los criterios vigentes respecto a la calidad de la educación en la universidad. 
'Assessment y Evaluation in Higher Education' es una revista internacional que desde 1975 publica comunicaciones y trabajos de investigación sobre todos los aspectos de evaluación en la Educación Superior. Su propósito es facilitar el acceso a información actualizada dentro del campo de la evaluación, compartir y hacer extensiva prácticas de evaluación innovadoras e ideas de significativo interés al respecto. Del total de artículos contabilizados en esta revista. los que abordan el tema de la evaluación del aprendizaje en la universidad resultaron más de la mitad. Es interesante señalar que en los últimos cinco años ha ido en ascenso el número de artículos sobre este tema.

La revista 'The Electronic Journal of Research in Educational Psychology (Revista Electrónica de Investigación Psicoeducativa (REIPE)', fundada en 2003, divulga investigaciones básicas y aplicaciones prácticas - reflexiones, experiencias profesionales, materiales de innovación tecnológica, material didáctico, material de evaluación e intervención - del ámbito de la Psicología Educativa y de la Psicopedagogía. En la actualidad edita tres números al año y en poco tiempo se ha convertido en una fuente de creciente interés. A pesar de la diferencia de su historial con relación a las otras fuentes seleccionadas, esta revista permite valorar el alcance y tratamiento que recibe el tema de la evaluación del aprendizaje universitario en una publicación de nuestro contexto. En esta fuente llama la atención el énfasis en la repercusión de la evaluación en el proceso de enseñanza y de aprendizaje, con atención a las voces de docentes y estudiantes.

\section{Unidades de análisis}

La revisión global del contenido de los artículos seleccionados sugirió focalizar tres cuestiones claves, suficientemente representativas de los temas tratados. La primera sobre el marco conceptual de la evaluación (sentido y significado); la segunda sobre los criterios y/o estándares para evaluar el aprendizaje y la tercera sobre métodos y procedimientos para evaluar. Estas temáticas constituyen las unidades de análisis para estructurar las reflexiones sobre los contenidos de los artículos revisados. Además se definen algunas categorías e indicadores que favorecieron la codificación y la valoración de las contribuciones analizadas. La tabla 2 contiene las unidades de análisis, las categorías y los indicadores definidos. 
Tabla. 2. Unidades, categorías e indicadores para el análisis del contenido

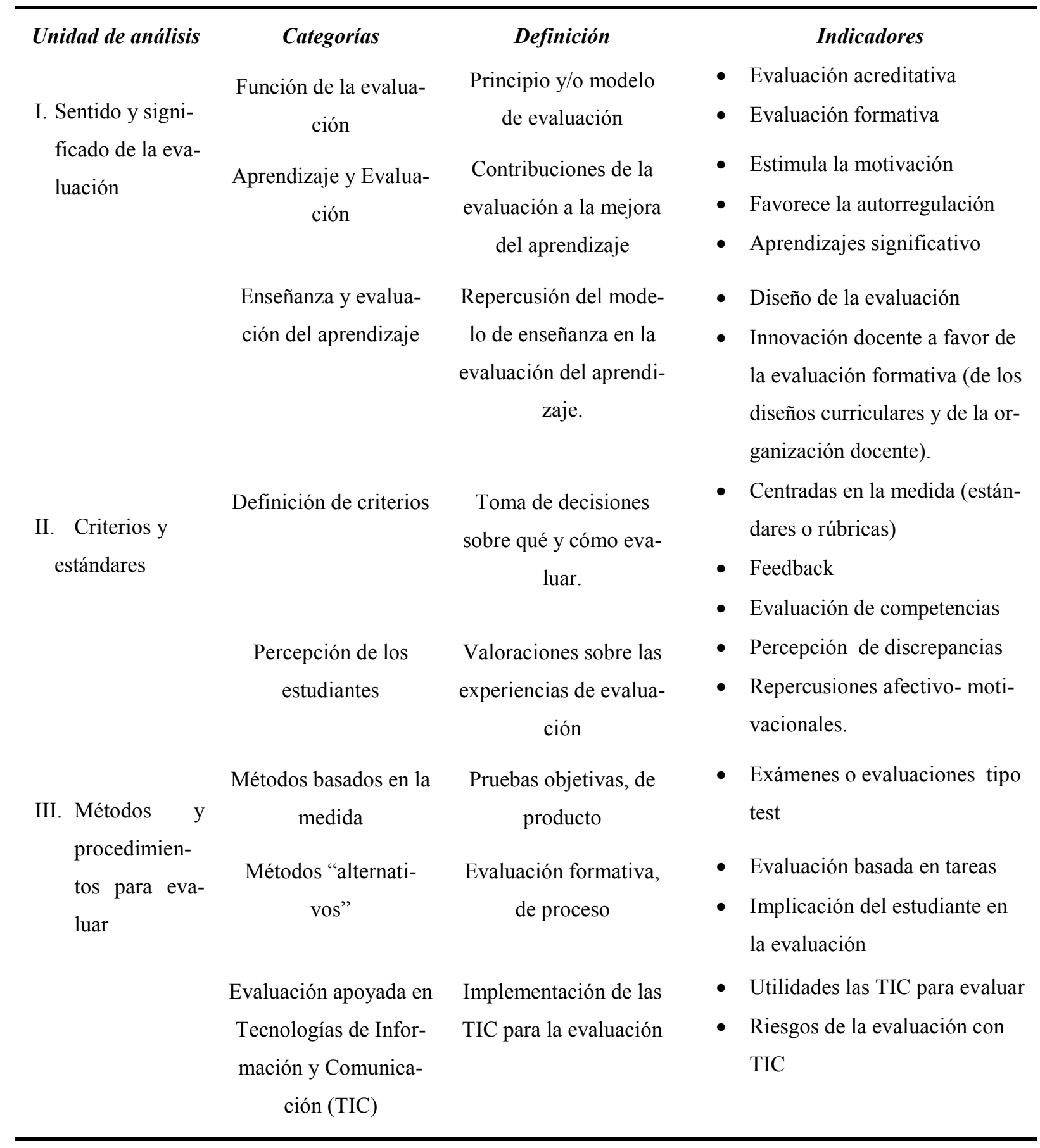

En las decisiones metodológicas de este estudio tomaron parte tres investigadoras que actualmente profundizan en este tema, dos de ellas en el marco del desarrollo de su tesis doctoral, la tercera su directora (autora de este artículo). La lista de referencias seleccionadas y sus resúmenes fue revisada sucesivamente por las investigadoras involucradas en el estudio, 
hasta conseguir suficiente acuerdo sobre la adecuación de las categorías e indicadores que servirían de soporte al análisis documental.

\section{Resultados}

A continuación se comentan, los resultados más significativos del análisis realizado. Para ordenarlos se agrupan alrededor de las unidades de análisis previamente definidas. El gráfico 1 resume la distribución de los artículos por unidades temáticas en las tres fuentes consultadas.

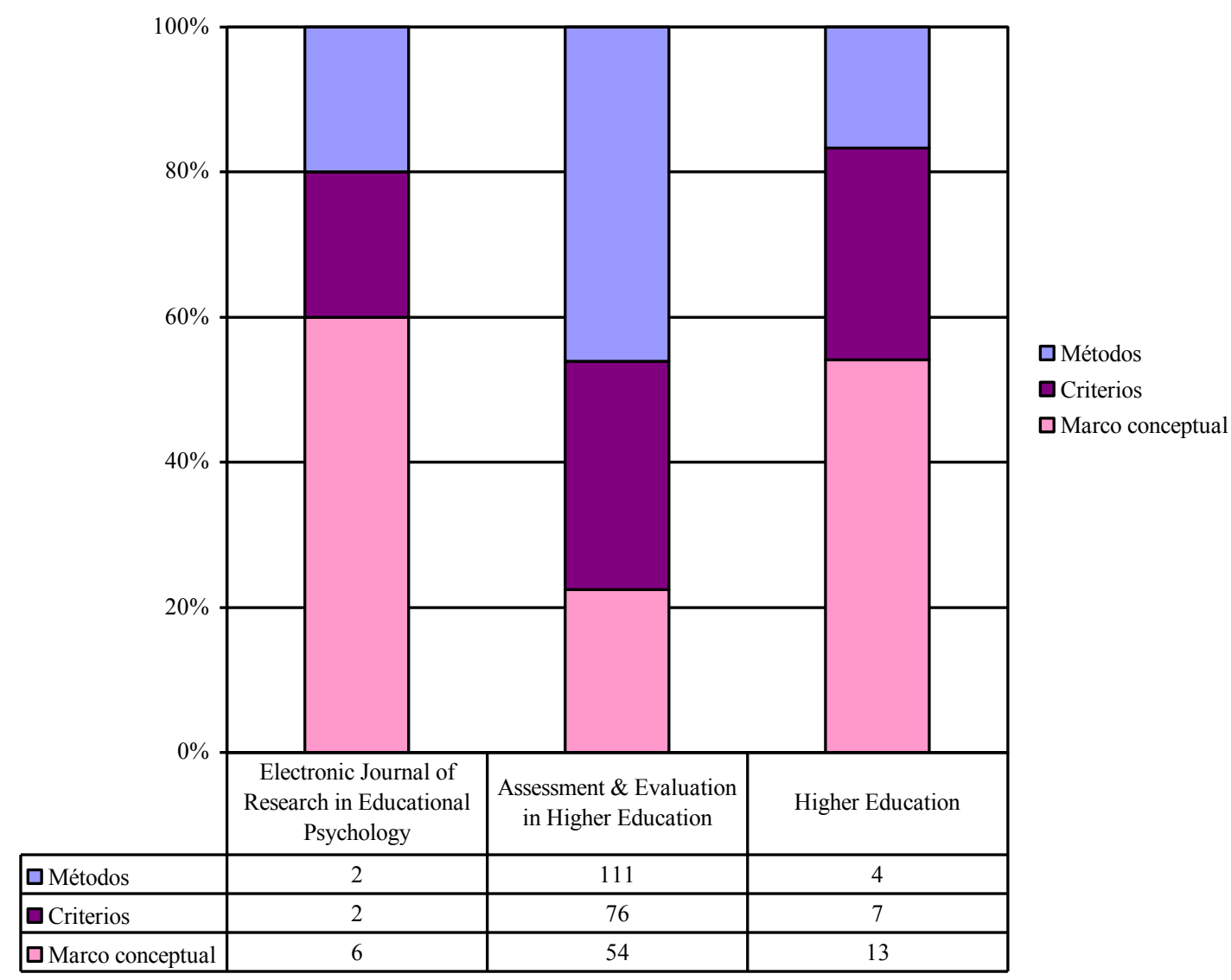

Gráfico 1. Proporción de artículos (\%) correspondientes a las unidades de análisis en las fuentes consultadas

Teniendo en cuenta el volumen de referencias categorizadas en el análisis que se presenta se citan, en cada tópico, solo aquellas que parecen más interesantes o que, incluso, según el criterio de la editorial de la revista, se consideran originales. También es intención mostrar a través de las referencias citadas la regularidad con que ha sido abordado el tema en cuestión a lo largo del período de tiempo delimitado para este análisis. 
Sentido y significado de la evaluación del aprendizaje en la universidad

Un primer y necesario análisis permite una aproximación al marco conceptual sobre el que giran las investigaciones sobre la evaluación del aprendizaje en la universidad, en concreto se valora el sentido y significado que se le atribuye. El $20 \%(n=56)$ de los artículos revisados fueron incluidos en esta unidad de análisis. La tabla 3 muestra la distribución de las referencias consultadas en este primer bloque atendiendo a estas tres subcategorías.

Tabla 3. Resumen de artículos referidos al marco conceptual de la evaluación

\begin{tabular}{lcc}
\hline \multicolumn{1}{c}{ Categorías } & Total de artículos & Por ciento \\
(1) Función de la evaluación & 19 & $33 \%$ \\
(2) Aprendizaje y Evaluación & 25 & $45 \%$ \\
(3) Enseñanza y evaluación del aprendizaje & 12 & $21 \%$ \\
\hline $\mathrm{N}=56$ & & \\
\end{tabular}

El análisis realizado, con foco en la función de la evaluación en el proceso de enseñanza y aprendizaje universitario, deja ver un cambio sustancial en este concepto desde finales de los 90. Desde entonces se ha mantenido un fuerte debate en torno a la efectividad de las fórmulas "tradicionales" de evaluación del aprendizaje, a la luz del acercamiento y respuestas de la universidad a los vertiginosos cambios sociales de finales de Siglo XX (Biggs, 1996).

En $33 \%$ de las referencias incluidas en esta primera unidad de análisis se constata una clara preocupación por distinguir y contrastar los principios de dos modelos de evaluación que se diferencian por su función, a veces contrapuestas: la evaluación tradicional o sumativa y la evaluación formativa. El análisis que se hace de los modelos de evaluación atiende sobre todo al impacto que tienen ambas metodologías en la calidad del aprendizaje de los estudiantes.

El propósito básico de la discusión en esta línea es la justificación o defensa de un modelo "alternativo" de evaluación que prioriza la función formativa (Dochy y McDowell, 1997; Yorke, 2003). Las investigaciones argumentan la necesidad de aproximar la evaluación del aprendizaje de los estudiantes universitarios al mundo laboral, con énfasis en la defensa 
del enfoque constructivista de la evaluación y del aprendizaje (Martin, 1997; Taylor y Marienau, 1997).

En los trabajos publicados en los años siguientes es notable el interés por demostrar la validez de denominada "evaluación alternativa", basada en el enfoque socio constructivista del aprendizaje. Numerosos estudios ratifican su validez y su positivo impacto en la calidad del aprendizaje de los estudiantes (Gosling, 2000; Kempner y Taylor, 1998; Rust, O'Donovan y Price, 2005; Walsh, 2007).

La vigencia de esta preocupación y la insistencia en la promoción del modelo de evaluación enfocado a la mejora del aprendizaje queda demostrada en la reciente dedicación de un número especial de la revista ‘Assessment y Evaluation in Higher Education' a este tópico - Special Issue: Learning-oriented assessment: principles and practice, 2006, 31(4).

En estrecha relación con este panorama conceptual en torno a las bases conceptuales de la evaluación, se profundiza en la naturaleza del aprendizaje deseable para el contexto universitario. En general estos trabajos se proponen ofrecer guías metodológicas que permitan poner la evaluación en función del aprendizaje y de su mejora, especialmente promover la autorregulación (Torrano y González, 2004; Van Eekelen, Boshuizen y Vermunt, 2005; Wilson y Scalise, 2006).

Buena parte de los trabajos categorizados en este primer bloque fundamentan la necesidad de estimular la participación de los estudiantes en las tareas de evaluación; el trabajo de Boud y Falchikov (2006) es excelente referencia en este sentido. Desde diversas perspectivas, se señala que la evaluación puede ser una valiosa oportunidad para motivar, mejorar y consolidar el aprendizaje, favoreciendo así el aprendizaje a lo largo de la vida y para la vida.

Por otra lado, se subraya la necesidad de conseguir cambios sustanciales en los modelos organizativos de nuestras universidades (Jackson y Ward, 2004). Es probable que esta preocupación explique la abundante publicación de experiencias educativas (estudio de casos) que apoyan la necesidad de revisar el modelo de enseñanza universitario atendiendo a sus repercusiones en la evaluación del aprendizaje. 
Evidencia esta preocupación el significativo número de investigaciones de las clasificadas en este bloque que se refieren a innovaciones docentes a favor de la evaluación (Leathwood y Phillips, 2000). Principalmente se aboga por cambios en el modelo educativo desde el nivel de la clase al currículo y cambios en la organización del equipo docente (Cook, 2001; McCormack, 2005; Sambell y McDowell, 1998; Sander, 2005; Stone y Friedman, 2002; Yorke, 1998).

Vale la pena subrayar que estas reflexiones teóricas no han estado exentas de debate y han generado muchísimas dudas. Los títulos de los trabajos siguientes, listados en orden cronológico, evidencian esta incertidumbre:

- Reflection: can we assess it? Should we assess it? (Sumsion y Fleet, 1996)

- Thinking Strategically about Assessment (Mutch, 2002)

- What is happening when we assess, and how can we use our understanding of this to improve assessment? (Baume, Yorke y Coffey 2004).

- How convincing is alternative assessment for use in higher education? (Maclellan, 2004).

- Do assessment methods matter? A sensitivity test (Tian, 2007).

En síntesis, sobre este primer tópico las investigaciones sucesivamente refuerzan la necesidad de instrumentar la evaluación de modo que facilite la mejora del aprendizaje. A raíz de éste énfasis en los últimos años se ha elaborado el término "evaluación orientada al aprendizaje" (en ingles 'learning-oriented assessment') definido alrededor de tres cuestiones esenciales:

1. Plantear las tareas de evaluación como tareas de aprendizaje.

2. Involucrar a los estudiantes en la evaluación.

3. Ofrecer los resultados o retorno de la evaluación (feed forward) a modo de feedback.

La evaluación orientada al aprendizaje enfatiza la necesidad de la promoción del aprendizaje a través de la evaluación, ponderando la función formativa y el feedback sobre la función sumativa y la calificación (Alonso, Asencio, López, Carriedo y Rycheck, 2007; Carless, 2003; Gibbs y Simpson, 2003, Santos Guerra, 2003;). 
Las preocupaciones que motivan estos estudios demuestran que el debate sigue abierto y las soluciones pendientes de implementar. Aún cuando las conclusiones de todos estos estudios apuntan hacia la validación de este enfoque de la evaluación integrado en la enseñanza y acercando el aprendizaje a la práctica y a la vida, los docentes universitarios tenemos la palabra y el reto.

\section{Criterios y estándares para evaluar el aprendizaje en la universidad}

Otro conjunto de referencias que se destaca se refiere a los criterios y estándares para la evaluación (entiéndase calificación) del aprendizaje en la universidad.

En relación al problema que entraña la definición de los criterios para evaluar se subraya la dificultad y la responsabilidad que conlleva la toma de decisiones al respecto, debido a su repercusión socioeducativa (Brooker, Muller, Mylonas y Hansford, 1998; Price, 2005). La discusión a lo largo del tiempo se centra en qué debe entenderse como criterio de medida, es decir qué debe evaluarse, para indicar, con equidad y justicia, lo que el estudiante universitario ha aprendido.

Las investigaciones demuestran que no hay entendimiento común sobre el significado de los 'criterios' y se señalan las graves implicaciones que tiene este hecho para la práctica docente. Además de demostrar la falta de claridad respecto a la definición de los criterios, estándares o rúbricas y la consecuente subjetividad con la que se califica el aprendizaje, se señala que en general, los profesores universitarios ocultan o no hacen explícitos al estudiante los criterios en los que basan sus juicios sobre la calidad de sus trabajos (Darby, 2007; Woolf, 2004; Stowell, 2004;).

En los últimos años se defiende con fuerza la necesidad de trascender el enfoque de medida para la evaluación del aprendizaje. En este sentido la investigación apunta hacia la conveniencia de procurar un clima de enseñanza dinamizador del aprendizaje: aprendizaje activo, aprendizaje basado en problemas, aprendizaje situado, aprendizaje cooperativo, aprendizaje a través de proyectos, entornos de aprendizaje con soportes tecnológicos 'TIC', etc. En tales situaciones de aprendizaje y enseñanza la evaluación viene a ser un factor dinamizador; consecuentemente la evaluación basada en la medida resulta ineficaz (Elwood y Val Kle- 
nowski, 2002; González, 2003; Havnes, 2004; Wilson y Fowler 2005; Serrad y Markham, 2005).

Es por ello que, en contraste y como alternativa a las dificultades que se atribuyen a la evaluación del aprendizaje a través de calificaciones o criterios estándares, se reitera la necesidad de incrementar la función formativa de la evaluación sobre la acreditativa o sumativa. El término consensuado para referirse a este tipo de evaluación se conoce como 'feedback' y es notable el incremento de investigaciones al respecto en los últimos años. Incluso se distinguen algunos procedimientos de 'feedback' que parecen ser altamente efectivos como guías para mejorar el aprendizaje a través de la evaluación (Cooper, 2000; Weaver, 2006; Duncan, 2007). Los estudios además muestran la positiva valoración que hacen los estudiantes de este modo evaluar.

Varios trabajos durante esta década validan estas propuestas; también comprueban que involucrar a los estudiantes en la evaluación de los resultados de su aprendizaje (evaluación por pares, auto evaluación y coevaluación) es sustancialmente beneficioso para este propósito (Smyth, 2004; Schelfhout, Dochy y Janssens, 2004; Richardson, 2005; Taras, 2001, 2003).

El cuanto a la complejidad que supone la definición de criterios para la evaluación de algunos conocimientos y habilidades en el ámbito universitario se menciona un grupo de aprendizajes más difíciles de comprobar o "medir", por ejemplo los que integran las competencias comunicativas (Elander, Harrington, Norton, Robinson, y Reddy, 2006; Joughin, 1998; Kuisma, 1999; Webster, Pepper y Jenkins, 2000;). Asimismo se percibe dificultad para evaluar el componente actitudinal de las competencias (Dalziel, 1998; Cowdroy y de Graaff, 2005; Tractenberg, Chaterji y Haramati, 2007).

Particularmente problemático se percibe la "medida" de la evaluación del aprendizaje en tareas que suponen el trabajo cooperativo, a pesar de que tanto estudiantes como docentes considera la evaluación (feedback) que proviene de estos procedimientos como más conveniente en relación con su impacto en la mejora de la calidad de sus aprendizajes (Kniveton, 1996; Laybourn, Goldfinch, Graham, MacLeod, y Stewart, 2001; MacLellan, 2001; Miller, 2003; Smyth, 2004; Trotter, 2006). 
La investigación en este tema también ha demostrado el significativo desfase que se percibe en la evaluación (calificación) del aprendizaje a través de exámenes de diferentes tipos; en este sentido resulta más cuestionable la corrección y/o calificación que se realiza a través de preguntas abiertas (Downs, 2006). La evaluación de tipo sumativa, resultante de los exámenes tipo test, si bien puede ser más “objetiva” parece tener peor impacto sobre la actitud de los estudiantes debido al negativo efecto que puede acarrear a nivel cognitivo y afectivomotivacional (Birenbaum, 2007).

La insatisfacción que genera cualquier discrepancia, particularmente cuando los resultados son inferiores a los esperados, traen consigo alteraciones en el comportamiento de los estudiantes que se expresan como incomodidad e irritación y que, por lo general, provocan disminución de la autoestima y del sentimiento de autoeficacia, o lo que es peor, pérdida de la motivación y desinterés por el estudio (Burton, 2006).

El estudio realizado por Thomson y Falchikov en 1998, publicada con el sugerente título "Full on Until the Sun Comes Out: the effects of assessment on student approaches to studying”, ofrece convincentes conclusiones sobre este fenómeno educativo. Entrevistas a amplia muestra de estudiantes y docentes mostró las preferencias de los estudiantes por particulares tipos de evaluación y la percepción de falta de armonía entre los modos de enseñanza y aprendizaje y la evaluación. Además, el estudio comprueba la extendida suposición de que los estudiantes estudian con mayor o menor estrés, incluso con perspicacia, teniendo en cuenta sus experiencias y expectativas acerca de la evaluación.

Investigaciones recientes continúan demostrando el incuestionable efecto que tiene sobre las actitudes de los estudiantes en las tareas de estudios no sólo la calificación que reciben, sino la percepción que tienen del modo en que sus profesores evalúan su aprendizaje (Struyven, Dochy y Janssens 2005). De otro lado, es notable la preocupación por resolver las diferencias entre lo que la universidad acredita como conocimiento y los aprendizajes funcionales que los estudiantes ponen en práctica, antes y después de su paso por la universidad (Dickinson, 2000; Sullivan, 2002). Un estudio realizado en universidades británicas refleja la elevada sensibilidad de este tema y la necesidad de considerar este tipo de conocimiento en la evaluación (Chisholm y Davis, 2007). En nuestra opinión ésta es la discusión más útil si queremos poner la universidad al servicio de la sociedad y, sobre todo, si se pretende avanzar en 
los procesos de acreditación y homologación de los estudios universitarios internacionalmente.

La creciente necesidad de aprender durante toda la vida en las sociedades modernas aumenta también la necesidad de aprendizaje a lo largo de toda la vida profesional y la evaluación debe facilitarlo y reflejarlo con suficiente certeza. Este es un planteamiento ampliamente aceptado y una tarea inaplazable en nuestras universidades.

\section{Discusión en torno a los métodos para evaluar el aprendizaje}

El desarrollo alcanzado en el terreno educativo universitario en las últimas décadas plantea un serio desafío al modelo de medida que ha predominado tradicionalmente en las prácticas de evaluación. La tradición ha conducido a un énfasis excesivo en la prueba tipo test y el mayor reto que se plantea al modelo tradicional de evaluación está precisamente en su alcance para evaluar el aprendizaje que se deriva de un enfoque diferente de enseñanza; por ej. el aprendizaje basado en problemas. Como alternativa emerge un modelo de evaluación educativa, denominado 'modelo de juicios' (Hager y Butler, 1996). Los supuestos básicos, los rasgos y los usos apropiados de éstos dos modelos de evaluación, se contrastan sistemáticamente en la investigación científica a lo largo de los últimos años.

En principio, el carácter selectivo presente en la certificación de los estudios académicos ha impregnado, en buena medida, el tipo de práctica evaluativa universitaria centrada en la calificación. El análisis de la evaluación universitaria a lo largo de los años, demuestra que se mantiene una fuerte preocupación por la acreditación, basada en estándares y en evaluaciones "objetivas".

Los estudios que abordan este tema se preguntan sistemáticamente si son todos los procedimientos igualmente válidos para cumplir las funciones que se asignan a la evaluación del aprendizaje en la universidad y, en caso de constatar limitaciones tanto en las fórmulas tradicionales como en las denominadas alternativas, se exploran los factores que las explican y se sugieren medidas relacionadas con el diseño de la evaluación para adecuarlas.

Atendiendo al objetivo de este trabajo, no procede describir el catálogo de procedimientos de evaluación de los aprendizajes que emerge del análisis de las referencias. Parece más interesante clasificarlas en dos grandes categorías: métodos basadas en la medida del aprendizaje y métodos alternativos, tomando como criterio para esta clasificación la función 
que se atribuye a la evaluación del aprendizaje. De este modo, pueden distinguirse claramente dos conjuntos de procedimientos: los métodos basados en la medida del aprendizaje (pruebas objetivas o de evaluación de productos) y los métodos formativos o de evaluación de procesos.

a) Métodos basados en la medida del aprendizaje o pruebas objetivas

Sobre este modelo de evaluación se cuestionan fundamentalmente sus aspectos metodológicos, tanto de construcción de las pruebas como de la validez y fiabilidad de la medida del aprendizaje. Los hallazgos de las investigaciones relacionan las dificultades de este tipo de evaluación o exámenes con el modo en que se redactan los ítems, con su longitud y con los modos de administración (Burton, 2004, 2005, 2006). Para este tipo de prueba se expresan profundas críticas desde la perspectiva lingüística (Paxton, 2000).

Otra restricción importante es que no permite comprobar juicios personales y el límite que marca para la expresión de los estudiantes. Sin embargo, no se descarta su validez frente a otras procedimientos, como demuestra el reciente estudio de Bleske-Rechek, Zeus y Webb (2007). Actualmente es objeto de debate el alcance de este tipo de exámenes para la evaluación de aprendizajes denominados 'complejos' (Williams, 2006).

Por otra parte, concerniente a la defensa de estos procedimientos se pueden encontrar varios informes de investigaciones que sugieren como controlar los riesgos y superar las limitaciones que se le reconocen a las 'pruebas objetivas'. Por ejemplo, solicitar la justificación de la respuesta en los test de selección múltiple (Fellenz, 2004); flexibilizar las opciones de selección, permitiendo a los estudiantes tomar decisiones al respecto, por ej. dar la posibilidad de que los propios alumnos generen preguntas de respuestas cerradas (Weber, Frary y Cross, 1995) o aplicar modelado estadístico para pruebas de selección múltiple y verdaderas/falsas para reducir la resolución de la incertidumbre atribuible a adivinación (Burton y Miller, 1999).

En síntesis, las investigaciones divulgadas sobre el valor de las pruebas objetivas continúan afirmando su idoneidad para evaluar el aprendizaje en la universidad en la medida que permiten averiguar lo que el alumno conoce, lo que consideran importante y lo que han comprendido; siempre que se cuide la elaboración de los ítems y el formato general de la prueba. A la vez en este valor radica su limitación, en tanto que supone una preparación laboriosa que de no ser exitosa, puede condicionar negativamente el estudio. 
b) Evaluación alternativa

En contraposición con la evaluación tradicional se han identificado en los últimos años un conjunto de procedimientos que se denomina 'evaluación alternativa' para distinguirlos de la evaluación basada en pruebas objetivas. Este tipo de evaluación también se denomina evaluación auténtica en el sentido de que la evaluación del aprendizaje se realiza a través de tareas reales (Leach, Neutze y Zepke, 2001; Romer, 2002; Welsh, 2002).

Ordenados por la frecuencia, los métodos más citados son: evaluación en el trabajo asociado al modelo de aprendizaje basado en el trabajo, en inglés Work-based learning (WBL), evaluación a través de proyectos, diarios para la evaluación del aprendizaje, evaluación por portafolios, metáforas y mapas conceptuales para la evaluación. Estos métodos tienen en común que las tareas de evaluación requieren que los estudiantes, en un determinado momento, demuestren, construyan, desarrollen un producto o solución a partir de unas definidas condiciones y estándares.

Los métodos que involucran a los estudiantes en la toma de decisiones sobre la evaluación son otro peculiar subconjunto que, atendiendo a estos principios también pueden considerarse como procedimientos alternativos para la evaluación. En la tabla 4 se presentan las frecuencias en que aparecen referidos en la consulta realizada.

Tabla 4. Resumen de artículos referidos a la evaluación alternativa

Métodos y procedimientos

Evaluación en el trabajo

Evaluación a través de proyectos

Diarios para la evaluación del aprendizaje

Evaluación por portafolios

Metáforas y mapas conceptuales

Evaluación con protagonismo de los estudiantes (evaluación por pares, autoevaluación y coevaluación)
Total de artículos

Por ciento

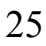

$26 \%$

9

$9 \%$

8

$8 \%$

4

$5 \%$

3

$4 \%$

46

$48 \%$ 
Sin duda alguna, el profesorado universitario, de manera más o menos intuitiva, tiene interiorizada la lógica del proceso de evaluación; la problemática emerge cuando en la toma de decisiones sobre qué y cómo evaluar carece de fundamentos o presenta significativa variabilidad. Esta circunstancia produce desorientación, tanto en el alumno como en la propia institución acerca de la validez y fiabilidad de la evaluación del aprendizaje y de los reales resultados de la formación profesional en la universidad. Muy brevemente, a continuación se acotan algunas cuestiones distintivas sobre la investigación en esta línea en cada uno de los métodos referenciados.

El aprendizaje basado en el trabajo (WBL), ha empezado a implementarse en una amplia variedad de contextos de enseñanza superior y cada vez es más valorado como método de enseñanza y aprendizaje universitario tanto en grado como postgrado (Forrester-Jones y Hatzidimitriadou, 2006; Gingerich, Kaye y Bailey, 1999; Toohey, 2002; Winter, 1994). Este método esencialmente se refiere al aprendizaje basado en la experiencia (Costley, 2007).

El interés que ha despertado y la importancia que se le atribuye queda reflejado en la dedicación de un número especial a este tema recientemente en la revista Assessment in Higher Education - Special Issue: Work-based learning: assessment and evaluation in higher education, 2007, 32(1). Sin embargo, el desarrollo de un enfoque pedagógico riguroso para fundamentar el WBL y su evaluación es todavía embrionario (Brodie y Irving, 2007).

Los resultados de los estudios sobre la evaluación basada en proyectos indican que este tipo de tareas de evaluación tiene una influencia positiva en el aprendizaje global (Frank y Barzilai, 2004; Gijbels, van de Watering y Dochy, 2005; Winning, Lim y Townsend, 2005). Estudiantes y profesores perciben ventajas de estas tareas de evaluación en relación con otros procedimientos.

A pesar de la incipiente utilización de estos métodos es importante considerar su implementación, en tanto el cambio que causa en los resultados del aprendizaje de los estudiantes es relativamente grande.

La utilización de los diarios para la evaluación del aprendizaje es más desconocida que otros métodos de este grupo. Sin embargo los estudios acerca de su validez demuestran que utilizados con frecuencia pueden desarrollar la reflexión crítica, sobre todo en relación 
con la práctica profesional, por ejemplo en la formación de profesores (O'Rourke, 1998). Las experiencias docentes a las que hace referencia su utilización sostienen un enfoque de la evaluación centrada en el aprendizaje (Crème, 2005; O'Connell y Dyment, 2006; Wagner, 1999; Woodward, 1998).

Las investigaciones que informan el uso de carpetas o portafolio para evaluar el aprendizaje reconocen que es un método ampliamente utilizado y que existe abundante literatura al respecto (Smith y Tillema, 2003). El valor de este método radica en su potencial contribución a la enseñanza y al aprendizaje reflexivo. Sin embargo se reconoce que la investigación carece de recomendaciones prácticas acerca de como puede ser utilizado el portafolio para desarrollar la comprensión del aprendizaje propio, de cómo puede evaluarse esta reflexión crítica sobre el aprendizaje y sobre la práctica profesional. Más recientemente comienza a divulgarse la utilización de este método con soporte tecnológico - portafolio digital (Woodward y Nanlohy, 2004).

Las conclusiones de las investigaciones coinciden en destacar algunas cuestiones clave sobre el uso del portafolio para la evaluación: la importancia de establecer el objetivo del portafolio; el positivo impacto del portafolio en la mejora del aprendizaje y en el desarrollo profesional de los estudiantes universitarios; los cambios que se aprecian en las prácticas profesionales a consecuencia del aprendizaje basado en la realización del portafolio y por último la necesidad de considerar cuestiones relacionadas con ética y confidencialidad cuando se implementa este procedimiento (Klenowski, Askew y Carnell, 2006).

Otro interesante método utilizado desde el enfoque de la evaluación hacia la mejora del aprendizaje son las metáforas. Una metáfora consiste en el uso de una palabra con un significado o en un contexto diferentes del habitual, en situaciones de aprendizaje se utilizan metáforas para comunicar conceptos abstractos de una forma familiar, 'abrir brechas' en el proceso de evaluación para permitir a los estudiantes articular su aprendizaje con las experiencias (Kemp, 1999). Los estudios científicos sobre su validez comprueban que puede ser tanto o más efectiva que otros métodos para dar cuenta del aprendizaje y particularmente para facilitar su construcción a través de la propia tarea de evaluación (Starr-Glass, 2005).

Por último, se analizan en este conjunto de 'métodos alternativos' los procedimientos de evaluación que tienen en cuenta al alumno: autoevaluación, evaluación por pares y coeva- 
luación. Estos métodos se diferencian entre sí por el rol que se le atribuye al estudiante, particularmente por su responsabilidad en la evaluación del aprendizaje. De los 46 trabajos que abordan estos procedimientos el $70 \%(\mathrm{n}=32)$ se refiere a la evaluación por pares, un $23 \%$ a la $(n=10)$ autoevaluación y el resto, 7\% $(n=4)$, a la coevaluación.

Boud y Falchikov (1989) analizan estudios publicados sobre estos procedimientos desde 1932 y su conclusión, más allá de los aspectos técnicos de análisis (fiabilidad, contenido, instrumentación, efectos, etc.), defiende la conveniencia de su implementación en un contexto de enseñanza en el que el aprendizaje del alumno, y no la docencia del profesor, debe ser el elemento central.

Sin embargo, en el ámbito universitario, plantear el tema de que el propio alumno sea juez y parte de la evaluación del aprendizaje puede correr el riesgo de un rechazo frontal por parte de cierto sector del profesorado. Pero, por una parte, las limitaciones de la evaluación centrada en el profesor que manifiestan los estudios arriba citados y por otra las potencialidades de estos métodos para estimular el aprendizaje profundo y autorregulado (Cassidy, 2007; Kirby y Downs, 2007;), han conllevado a que los procedimientos que involucran al alumno en la evaluación comiencen a ser más utilizados en los últimos años (Cheng, 2001; Carey y Gregory, 2003; De la Fuente y Justicia, 2003; Jones, Laufgraben y Morris, 2006; Lundberg, 2004; Silén, 2006; Taras 2002).

Este tipo de evaluación parece ser más empleada en situaciones de trabajo en grupos (Bushell, 2006; Conway, Kember, Sivan y Wu, 1993; Freeman, 1995; Goldfinch, 1994; Houston y Lawrence, 2001; Lazenbatt, 1996; Lejk, Wyvill y Farrow, 1997; Lejk y Wyvill, 2001, 2002; Magin, 2001; Somervell, 1993). Asimismo se publican interesantes estudios que involucran, además de los estudiantes, a la empresa o a potenciales empleadores (Rainsbury, Hodges, Sutherland y Barrow, 1998).

En relación con estos métodos dos nuevos términos han surgido en la literatura y en la práctica sobre la evaluación del aprendizaje: co-assessment y peer review (Dancer y Kamvounias, 2005; Gale, Martin y McQueen, 2002; Giles, Martin, Bryce y Hendry, 2004; Stefani, 1998). La diferencia entre éstos y los ya conocidos estriba en que en este caso la calificación es generada por el profesor, a partir de unos determinados criterios pactados, y son los estudiantes quienes ponderan esa calificación dentro de cada uno de los miembros del grupo 
de trabajo. Los beneficios derivados de este procedimiento de evaluación son sintetizados en la revisión de publicaciones al respecto realizada por Topping (1998).

Algunos estudios prueban la eficacia de la evaluación que involucra a los estudiantes en grupos de clase numerosos (Ballantyne, Hughes y Mylonas, 2002). Especialmente útil parece ser este procedimiento para evaluar durante presentaciones orales (Cheng y Warren, 1999; Langan, et al., 2005; MacAlpine, 1999). En general, los estudiantes expresan satisfacción con el uso de estos procedimientos y se comprueba su positivo efecto en la mejora de la calidad del aprendizaje (Gatfield, Bisset, Borja, Brassard, Reohr, O'Neill y Kosky, 1999).

\section{Evaluación a través de las tecnologías de la información y comunicación (TICs)}

A propósito de este auge en la revisión realizada para esta comunicación, $23 \%(n=22)$ de los artículos publicados fueron clasificados en la categoría 'métodos de evaluación que utilizan las TICs. Estos estudios valoran ventajas y desventajas de los contextos educativos que utilizan las TICs para instrumentar diferentes procedimientos de evaluación del aprendizaje tanto tradicional (basada en pruebas objetivas) como los denominados 'métodos alternativos'.

Las TICs conllevan a disponer en red de un volumen enorme, casi ilimitado, de información dinámica y cambiante. En la era de la llamada sociedad de la información y del conocimiento, cobra especial importancia la enseñanza y el aprendizaje mediado por TICs: a distancia, virtual, etc., y esto trae consigo, también, inevitables innovaciones en los sistemas de evaluación.

Desde finales de los años 90 se constata masiva implementación de las TICs para la evaluación (Zakrzewski y Bull, 1998; Welsh, Alexander y Dey, 2001). Éstas pueden ser herramientas útiles para la evaluación de los aprendizajes en la medida que facilitan la recogida, valoración y devolución de información a los estudiantes sobre su proceso de aprendizaje. A la vez pueden promover la autorregulación y mejoran el aprendizaje (Gamliel y Davidovitz, 2005; Challis, 2005; Macdonald, 2004).

En los últimos años se aprecia una amplia utilización de diversos procedimientos de evaluación en entornos de enseñanza y aprendizaje universitarios con soportes TICs [CSCL 
environment] considerando la participación de los estudiantes en la evaluación (Prins, Sluijsmans, Kirschner y Strijbos, 2005; Keppell, Au y Chan, 2006).

El estudio realizado por Watt, Simpson, McKillop y Nunn (2002) muestra las ventajas que ofrecen las TICs para automatizar el feedback y en este mismo sentido son bastante populares los software que permiten a los docentes elaborar rúbricas online (Campbell, 2005). Resultados bastante útiles de estos estudios ofrecen guías para desarrollar la evaluación con TIC (Coll, Rochera, Mayordomo y Naranjo, 2007; Hosie, Schibeci y Backhaus, 2005).

- El lado oscuro de las TIC en la evaluación del aprendizaje

Por último, en el subconjunto de publicaciones clasificadas en la categoría que alude al impacto de las TIC en la evaluación del aprendizaje, muy a nuestro pesar, se hace necesario incluir una subcategoría que indica su desfavorablemente incidencia en la evaluación (Ricketts y Zakrzewski, 2005). Una buena parte de los estudios intenta averiguar las causas que conducen a los estudiantes al plagio (Bennett, 2005; Macdonald y Carroll, 2006; Park, 2003) y otro grupo de trabajos intenta controlarlo o impedirlo a través de la tecnología (Badge, Cann y Scott, 2007; Walden y Peacock, 2004). Estas investigaciones analizan el sentido y el contexto del plagio, el tipo de plagio que realizan los estudiantes, las actitudes ante el plagio y los desafíos que plantea el plagio digital. En todos los trabajos se subraya la necesidad de promover la integridad y la dignidad académica y de encontrar modos efectivos de evitar este negativo fenómeno.

Los estudios realizados hasta el momento han concluido que el plagio es indudablemente común y que su mayor incidencia se explica por el aumento del acceso a fuentes digitales, incluyendo Internet. Se reconocen motivos múltiples para que los estudiantes plagien, también relacionados con el formato de evaluación que se utiliza. A menudo los estudiantes racionalizan su comportamiento tramposo y la importancia del plagio es minimizada por ellos y por sus compañeros de clase.

McKeever (2006) apunta que a pesar de que el crecimiento exponencial del Internet haga más fácil que nunca el plagio, también hace mucho más fácil descubrirlo. Su trabajo describe diferentes métodos para descubrir el plagio en la Web, no obstante enfatiza en la necesidad de contar con la mejora de las actitudes de docentes y estudiantes, más que controlarlo por vías coercitivas. 


\section{Conclusiones y recomendaciones}

El análisis realizado sobre las tendencias de la investigación científica acerca del tema de la evaluación del aprendizaje en la universidad en las fuentes consultadas pone de relieve que en la última década son recurrentes las mismas preocupaciones. Los focos de atención son los tareas clásicas que incumben al docente a la hora de evaluar (1) Acotar las funciones de la evaluación; (2) Decidir sobre los métodos para evaluar el aprendizaje (3) Definir los criterios y estándares de modo válido, fiable, justo y equitativo.

Por una parte, se encuentran idénticas manifestaciones de los problemas abordados y por otra, cada vez más se coincide en las soluciones que se proponen. Paulatinamente, se está perfilando una nueva forma de entender la formación universitaria que re-conceptualiza la enseñanza y el aprendizaje, dando lugar a una cultura educativa. Al mismo tiempo se ha modificado considerablemente el enfoque de la evaluación originando una concepción 'alternativa' o una nueva 'cultura de la evaluación'.

La necesidad de evaluar no sólo los logros académicos sino también las competencias genéricas y transferibles a múltiples situaciones (perfomance-based assessment) no sólo ha puesto en crisis el uso de las tradicionales pruebas de evaluación, sino que ha hecho extensivo el uso de una evaluación auténtica.

La evaluación debe trascender el enfoque de medida y la constatación de la capacidad de reproducir el conocimiento que demandan las pruebas objetivas. Existe amplio consenso respecto a que las funciones de la evaluación van más allá de la comprobación del nivel de conocimientos alcanzados en una determinada asignatura y que en su lugar, la evaluación debe ser capaz de reflejar en qué medida los estudiantes son capaces de usar el conocimiento de manera interrelacionada al analizar y resolver problemas auténticos. Por ende, la práctica evaluativa en la universidad no puede continuar teniendo como referente a la asignatura y al profesorado (individualmente considerado).

De otro lado, queda demostrado que los procedimientos tradicionales de evaluación no satisfacen los requisitos que exige la evaluación del aprendizaje basado en competencias y en 
metodologías activas. Por ello, cada vez son más los estudios que defienden una re- conceptualización y una práctica de la evaluación que no rechaza los presupuestos de la evaluación tradicional, sino que los incluye en una propuesta complementaria que añade y potencia nuevos planteamientos.

Evidencias irrefutables de investigaciones en diferentes momentos y escenarios educativos, siguen advirtiendo que la naturaleza de las tareas de evaluación influye definitivamente en el enfoque que los estudiantes adoptan para aprender. En ese sentido los enfoques de evaluación tradicionales pueden tener efectos contrarios a los deseados.

En este sentido, desde propuestas diversas, actualmente se extiende un enfoque de la evaluación orientado a la mejora del aprendizaje. Los procedimientos que se consideran como 'métodos alternativos' a la evaluación tradicional contienen tareas reales o auténticas que conllevan soluciones reflexivas, en las que los estudiantes deben interpretar, analizar, evaluar problemas y explicar sus argumentos.

Más que la medida, la evaluación se concibe como parte del proceso de aprendizaje. Supone procedimientos que permitan la recogida de amplias evidencias o información sobre el aprendizaje que realiza el alumno y se considera que debe ser un proceso compartido y negociado por los diferentes agentes implicados en el mismo. La evaluación de esto modo favorece el carácter reflexivo y la autorregulación desde el principio hasta el final del proceso educativo.

En síntesis, las investigaciones de los últimos años hacen patente la necesidad de cambios en las prácticas de evaluación. Sin embargo, plantear una nueva cultura de la evaluación no se refiere, exclusivamente, a cambiar los métodos o instrumentos de evaluación sino, lo que es más importante, a cambiar la concepción que sobre este proceso tienen los docentes y los estudiantes universitarios.

El desafío de esta última década en relación con la calidad de la evaluación en la Educación Superior está en la necesidad de impregnar la evaluación de realismo; conseguir evaluar el aprendizaje de los estudiantes universitarios a través de tareas profesionales auténticas correlativas o próximas al practicum. 
Las conclusiones que el estudio realizado permite elaborar son sumamente limitadas, al ser extraídas del análisis de una pequeña parte de la literatura científica disponible sobre este tópico; en este sentido profundizar y ampliar esta indagación seguramente ofrece confirmaciones y nuevas pistas. No obstante, con los modestos resultados que se han podido alcanzar creemos haber contribuido en algo a sensibilizar a la comunidad educativa universitaria respecto a la necesidad de acometer el cambio que reclaman la evaluación del aprendizaje, al menos en las cuestiones que fueron acotadas. Como docentes, saber ver, analizar, reflexionar sobre todos los elementos que nos puede mostrar una buena evaluación permite acercarse a un proceso de mejora continua y de calidad; no sólo del proceso de enseñanza-aprendizaje sino del contexto educativo en el cual se desarrolla la educación superior.

La rápida mirada dada a la divulgación científica sobre la evaluación del aprendizaje en la universidad permite además una última recomendación. Teniendo en cuenta la regularidad y la insistencia con que vienen apareciendo los mismos tópicos abrir nuevas brechas en la investigación en este campo probablemente sea redundante. Parece más pertinente nutrirse de la amplia base de referencias que tenemos al alcance y proceder a la renovación de nuestras prácticas docentes.

\section{Referencias}

Alonso, J., Asencio, F., López, I., Carriedo, N., y Rycheck, F. (2007). Evaluación para aprender: Las ideas de los profesores de ciencias sociales sobre la evaluación del entendimiento causal. Electronic Journal of Research in Educational Psychology, 5(3), 593616.

Badge, J., Cann, A., y Scott, J. (2007). To cheat or not to cheat? A trial of the JISC Plagiarism Detection Service with biological sciences students. Assessment y Evaluation in Higher Education, 32(4), 433-439.

Ballantyne, R., Hughes, K., y Mylonas, A. (2002). Developing Procedures for Implementing Peer Assessment in Large Classes Using an Action Research Process. Assessment y Evaluation in Higher Education, 27(4), 427-441.

Baume, D., Yorke, M., y Coffey, M. (2004). What is happening when we assess, and how can we use our understanding of this to improve assessment?. Assessment y Evaluation in Higher Education, 29 (4), 451-477. 
Bennett, R. (2005). Factors associated with student plagiarism in a post-1992 university. Assessment y Evaluation in Higher Education, 30(2), 137-162.

Biggs, J. (1996). Assessing Learning Quality: reconciling institutional, staff and educational demands. Assessment y Evaluation in Higher Education, 21(1), 5-16.

Biggs, J. (2005). Calidad del aprendizaje universitario. Madrid: Narcea.

Billing. D. (2007). Teaching for transfer of core/key skills in higher education: Cognitive skills, Higher Education, 53(6), 483-516.

Birenbaum, M. (2007). Assessment and instruction preferences and their relationship with test anxiety and learning strategies. Higher Education, 53(6), 749-768.

Bleske-Rechek, A., Zeug, N., y Webb, R. (2007). Discrepant performance on multiplechoice and short answer assessments and the relation of performance to general scholastic aptitude. Assessment y Evaluation in Higher Education, 32 (2), 89-105.

Boud, D., y Falchikov N. (2006). Aligning assessment with long-term learning. Assessment y Evaluation in Higher Education, 31 (4), 399-413.

Boud, D., y Falchikov, N. (1989). Quantitative studies of self-assessment in higher education: a critical analysis of findings. Higher Education, 18, 529-549.

Broabfoot, P., y Black, P. (2004). Redefining assessment? The first ten years of Assessment in Education. Assessment in Education, 11(1), 1-27.

Brodie, P., y Irving, P. (2007). Assessment in work-based learning: investigating a pedagogical approach to enhance student learning. Assessment y Evaluation in Higher Education, 32(1), 11-19.

Brooker, R., Muller, R., Mylonas, A. y Hansford, B. (1998). Improving the Assessment of Practice Teaching: a criteria and standards framework. Assessment y Evaluation in Higher Education, 23(1), 5-24.

Brown, S., y Glasner, A. (2003): Evaluar en la Universidad. Problemas y nuevos enfoques. Madrid: Narcea.

Burton, N. (2006). Student justice perceptions following assignment feedback. Assessment y Evaluation in Higher Education, 31(6), 655-670.

Burton, R. (2005). Multiple-choice and true/false tests: myths and misapprehensions. Assessment y Evaluation in Higher Education, 30(1), 65-72.

Burton, R. (2004). Multiple choice and true/false tests: reliability measures and some implications of negative marking. Assessment y Evaluation in Higher Education, 29(5), $585-595$. 
Burton, R., y Miller, D. (1999). Statistical Modelling of Multiple-choice and True/False Tests: ways of considering, and of reducing, the uncertainties attributable to guessing. Assessment y Evaluation in Higher Education, 24 (4), 399-411.

Bushell, G. (2006). Moderation of peer assessment in group projects. Assessment y Evaluation in Higher Education, 31(1), 91-108.

Campbell, A. (2005). Application of ICT and rubrics to the assessment process where professional judgement is involved: the features of an e-marking tool. Assessment y Evaluation in Higher Education, 30(5), 529-537.

Carey, J. y Gregory, V. (2003). Toward Improving Student Learning: policy issues and design structures in course-level outcomes assessment. Assessment y Evaluation in Higher Education, 28 (3), 215-227.

Carless, D. (2003). Learning-oriented assessment. Paper presented at the Evaluation and Assessment Conference. Adelaide, University of South Australia, November, 25, 2003.

Cassidy, S. (2007). Assessing 'inexperienced' students' ability to self-assess: exploring links with learning style and academic personal control. Assessment y Evaluation in Higher Education, 32 (3), 313-330

Challis, D. (2005). Committing to quality learning through adaptive online assessment. Assessment y Evaluation in Higher Education, 30(5), 519-527.

Cheng, D. (2001). Assessing Student Collegiate Experience: where do we begin?. Assessment y Evaluation in Higher Education, 26(6), 525-538.

Cheng, W y Warren, M. (1999). Peer and Teacher Assessment of the Oral and Written Tasks of a Group Project. Assessment y Evaluation in Higher Education, 24(3) 301-314.

Chisholm, C., y Davis, M. (2007). Analysis and evaluation of factors relating to accrediting $100 \%$ of prior experiential learning in UK work-based awards. Assessment y Evaluation in Higher Education, 32(1), 45-59.

Coll, C., Rochera, Ma . J., Mayordomo, R. y Naranjo, M. (2007). Coll, Evaluación continua y ayuda al prendizaje. Una experiencia de innovación en Educación Superior con ayuda de TIC. Electronic Journal of Research in Educational Psychology, 5(3), 783-804.

Conway, R, Kember, D., y Sivan, A., WU, M. (1993). Peer Assessment of an Individual 's Contribution to a Group Project. Assessment y Evaluation in Higher Education, 18(1), 45-56.

Cook, A. (2001). Assessing the Use of Flexible Assessment. Assessment y Evaluation in Higher Education, 26(6), 539-549. 
Cooper, N. (2000). Facilitating Learning from Formative Feedback in Level 3 Assessment. Assessment y Evaluation in Higher Education, 25 (3).279-291.

Costley, C. (2007). Work-based learning: assessment and evaluation in higher education. Assessment y Evaluation in Higher Education, 32 (1), 1-9.

Cowdroy, R., y de Graaff, E.(2005). Assessing highly-creative ability. Assessment y Evaluation in Higher Education, 30(5), 507-518

Crème, Ph. (2005). Should student learning journals be assessed?. Assessment y Evaluation in Higher Education, 30(3), 287-296.

Dalziel, J. (1998). Using Marks to Assess Student Performance, some problems and alternatives. Assessment y Evaluation in Higher Education, 23(4), 351-366.

Dancer, D., y Kamvounias, P. (2005). Student involvement in assessment: a project designed to assess class participation fairly and reliably. Assessment y Evaluation in Higher Education, 30(5), 445-454.

Darby, J. (2007). Evaluating course evaluations: the need to establish what is being measured. Assessment y Evaluation in Higher Education, 32 (4), 441-455.

De la Fuente, J. y Justicia, F. (2003). Escala de Estrategias de Aprendizaje ACRA- Abreviada para Alumnos Universitarios. Electronic Journal of Research in Educational Psychology, 1(2), 140-158.

Dickinson, M. (2000). An Analysis of the Accreditation of Transferable Skills in Extra Curricular Activities within Higher Education. Assessment y Evaluation in Higher Education, 25 (1), 61-70.

Dochy, F., y McDowell, L. (1997). Introduction: Assessment as a tool for learning. Studies in Educational Evaluation, 23, 279-298.

Downs, C. (2006). What should make up a final mark for a course? An investigation into the academic performance of first year Bioscience students. Assessment y Evaluation in Higher Education, 31 (3), 345-364.

Duncan, N. (2007). 'Feed-forward': improving students' use of tutors' comments. Assessment y Evaluation in Higher Education, 32(3), 271-283.

Elander, J., Harrington, K, Norton, L., Robinson, H. y Reddy, P. (2006). Complex skills and academic writing: a review of evidence about the types of learning required to meet core assessment criteria. Assessment y Evaluation in Higher Education, 31(6), 71-90.

Elwood, J., y Val Klenowski (2002). Creating Communities of Shared Practice: the challenges of assessment use in learning and teaching. Assessment y Evaluation in Higher Education, 27(3), 243-256. 
Fellenz, M. (2004). Using assessment to support higher level learning: the multiple choice item development assignment. Assessment y Evaluation in Higher Education, 29(6), 703-719.

Forrester-Jones, R., y Hatzidimitriadou, E. (2006). Learning in the real world? Exploring widening participation student views concerning the 'fit' between knowledge learnt and work practices. Assessment y Evaluation in Higher Education, 31 (6), 611-624.

Freeman, M. (1995). Peer Assessment by Groups of Group Work. Assessment y Evaluation in Higher Education, 20(3), 289-300.

Gale, K., Martin, K., y McQueen, G. (2002). Triadic Assessment. Assessment y Evaluation in Higher Education, 27(6), 557-567.

Gamliel, E., y Davidovitz, L. (2005). Online versus traditional teaching evaluation: mode can matter. Assessment y Evaluation in Higher Education, 30 (6), 581-592.

Gatfield, T., Bisset, J., Borja, M., Brassard, D., Reohr, J., O'Neill, K., y Kosky, R. (1999). Examining Student Satisfaction with Group Projects and Peer Assessment. Assessment y Evaluation in Higher Education, 24 (4), 365-377.

Gibbs, G. y Simpson, C. (2003). Does your assessment support your students' learning?. Retrieved March 11, 2008, from http://arts.unitec.ac.nz/documents/GrahamGibbAssessmentLearning.pdf

Gijbels, D., van de Watering, G., y Dochy, F. (2005). Integrating assessment tasks in a problem-based learning environment. Assessment y Evaluation in Higher Education, 30 (1), 73-86.

Giles, A., Martin, S., Bryce, D. y Hendry, G. (2004). Students as partners in evaluation: student and teacher perspectives. Assessment y Evaluation in Higher Education, 29(6), 681-685.

Gingerich, W., Kaye, K., y Bailey, D. (1999). Assessing Quality in Social Work Education: focus on diversity. Assessment y Evaluation in Higher Education, 18 (2), 119-129.

González, I. (2006). Dimensiones de la evaluación en el Espacio Europeo de Educación Superior. Electronic Journal of Research in Educational Psychology. 4 (3). 445-468.

González, L. (2003). Aproximación a una formación académica de calidad: el punto de vista de los estudiantes universitarios. Electronic Journal of Research in Educational Psychology, 1(1), 20-42.

Gosling, D. (2000). Using Habermas to Evaluate Two Approaches to Negotiated Assessment. . Assessment y Evaluation in Higher Education, 25(3), 293-304. 
Hager, P., y Butler, J. (1996). Two Models of Educational Assessment. Assessment y Evaluation in Higher Education, 21 (4), 367-378.

Havnes, A. (2004). Examination and learning: an activity-theoretical analysis of the relationship between assessment and educational practice. Assessment y Evaluation in Higher Education, 29(2), 159-176.

Hosie, P., Schibeci, R., y Backhaus, A. (2005). A framework and checklists for evaluating online learning in higher education. Assessment y Evaluation in Higher Education, 30 (5), 539-553.

Houston K., y Lazenbatt, A. (1996). A Peer-tutoring Scheme to Support Independent Learning and Group Project Work in Mathematics. Assessment y Evaluation in Higher Education, 21(3), 251-266.

Jackson, N., y Ward, R. (2004). A fresh perspective on progress files - a way of representing complex learning and achievement in higher education. Assessment y Evaluation in Higher Education, 29(4), 423-449.

Jones, P, Laufgraben, J., y Morris, N. (2006). Developing an empirically based typology of attitudes of entering students toward participation in learning communities. Assessment y Evaluation in Higher Education, 31 (5), 249-265.

Jongbloed, B. (2002). Lifelong learning: Implications for institutions. Higher Education, 44 (3/4), 413-431.

Joughin, G. (1998). Dimensions of Oral Assessment . Assessment y Evaluation in Higher Education, 23(4), 367-378.

Kemp, E. (1999). Metaphor as a Tool for Evaluation. Assessment y Evaluation in Higher Education, 24(1), 81-89.

Kempner, K. y Taylor, C. (1998). An alternative assessment to higher education outcomes: Differentiating by institutional type. Higher Education, 36(3), 301-321.

Keppell, M., Au, E., y Chan, C. (2006). Peer learning and learning-oriented assessment in technology-enhanced environments. Assessment y Evaluation in Higher Education, $31(4), 453-464$.

Kirby, N., y Downs, C. (2007). Self-assessment and the disadvantaged student: potential for encouraging self-regulated learning? . Assessment y Evaluation in Higher Education, 32(4), 475-494.

Klenowski, V., Askew, S., y Carnell, E. (2006). Portfolios for learning, assessment and professional development in higher education. Assessment y Evaluation in Higher Education, 3 (3), 267-286 
Kniveton, B. (1996). Student Perceptions of Assessment Methods. Assessment y Evaluation in Higher Education, 21 (3), 229-237.

Kuisma, R. (1999). Criteria Referenced Marking of Written Assignments. Assessment y Evaluation in Higher Education, 24 (1), 27-39.

Langan, M., Wheater, Ph., Shaw, E., Haines, B., Cullen, R., Boyle, J., Penney, D., Oldekop, J., Ashcroft, C., Lockey, L., y Preziosi, R. (2005). Peer assessment of oral presentations: effects of student gender, university affiliation and participation in the development of assessment criteria. Assessment y Evaluation in Higher Education, 30(1), 2134.

Lawrence, K. (2001). Some Refinements on Peer Assessment of Group Projects. Assessment y Evaluation in Higher Education, 26(1), 5-18.

Laybourn, Ph., Goldfinch, J., Graham, J., MacLeod, L., y Stewart, Sh. (2001). Measuring changes in groupworking skills in undergraduate students after employer involvement in group skill development. Assessment y Evaluation in Higher Education, 26(4), 367380 .

Leach, L., Neutze, G., y Zepke, N. (2001). Assessment and Empowerment: some critical questions. Assessment y Evaluation in Higher Education, 26(4), 293-305.

Leathwood, C. y Phillips, D. (2000). Developing curriculum evaluation research in higher education: Process, politics and practicalities. Higher Education, 40(3), 293-311.

Lejk, M., y Wyvill, M. (2001). Peer assessment of contributions to a group project: a comparison of holistic and category-based approaches. Assessment y Evaluation in Higher Education, 26(1), 61-72.

Lejk, M., Wyvill, M., y Farrow, S. (1997). Group learning and group assessment on undergraduate computing courses in Higher Education in the UK: results of a survey. Assessment y Evaluation in Higher Education, 22(1), 81-91.

Lundberg, A. (2004). Student and teacher experiences of assessing different levels of understanding. Assessment y Evaluation in Higher Education, 29(3), 323-333.

MacAlpine, J. (1999). Improving and encouraging peer assessment of student presentations . Assessment y Evaluation in Higher Education, 24(1), 15-25.

Macdonald, J. (2004). Developing competent e-learners: the role of assessment. Assessment $y$ Evaluation in Higher Education, 29(2), 215-226.

Macdonald, R., y Carroll, J. (2006). Plagiarism - a complex issue requiring a holistic institutional approach. Assessment y Evaluation in Higher Education, 31 (2), 233-245. 
MacLellan, E. (2001). Assessment for Learning: the differing perceptions of tutors and students. Assessment y Evaluation in Higher Education, 26(4), 307-318.

Maclellan, E. (2004). How convincing is alternative assessment for use in higher education?. Assessment y Evaluation in Higher Education, 29(3), 311-321.

Magin, D. (2001). A novel technique for comparing the reliability of multiple peer assessments with that of single teacher assessments of group process work. Assessment $y$ Evaluation in Higher Education, 26(2), 139-152.

Martin, S. (1997). Two models of educational assessment: a response from initial teacher education: if the cap fits. Assessment y Evaluation in Higher Education, 22(3), 37-343.

McCormack, C. (2005). Reconceptualizing student evaluation of teaching: an ethical framework for changing times. Assessment y Evaluation in Higher Education, 30(5), 463476.

McKeever, L. (2006). Online plagiarism detection services - saviour or scourge?. Assessment y Evaluation in Higher Education, 31 (2), 155-165.

Miller, P. (2003). The effect of scoring criteria specificity on peer and self-assessment. Assessment y Evaluation in Higher Education, 28(4), 383-394.

Mollis, M.,. y Marginson, S. (2002). The assessment of universities in Argentina and Australia: Between autonomy and heteronomy. Higher Education, 43(3), 311-330.

Mutch, A. (2002). Thinking strategically about assessment. Assessment y Evaluation in Higher Education, 27(2), 163-174.

O'Connell, T., y Dyment, J. (2006). Reflections on using journals in higher education: a focus group discussion with faculty. Assessment y Evaluation in Higher Education, 31 (6), $671-691$

O'Rourke, R. (1998). The Learning Journal: from chaos to coherence. Assessment y Evaluation in Higher Education, 23(4), 403-413.

Park, Ch. (2003). In Other (People's) Words: plagiarism by university students--literature and lessons. Assessment y Evaluation in Higher Education, 28(5), 471-488.

Paxton, M. (2000). A linguistic perspective on multiple choice questioning. Assessment y Evaluation in Higher Education, 25 (2) 109-119.

Price, M. (2005). Assessment standards: the role of communities of practice and the scholarship of assessment. Assessment y Evaluation in Higher Education, 30 (3), 215-230.

Prins, F., Sluijsmans, D., Kirschner, P., y Strijbos, J. (2005). Formative peer assessment in a CSCL environment: a case study. Assessment y Evaluation in Higher Education, 30 (4), 417-444. 
Rainsbury, E., Hodges, D., Sutherland, J., y Barrow, M. (1998). Academic, employer and student collaborative aAssessment in a work-based cooperative education course. $A s$ sessment y Evaluation in Higher Education, 23(3), 313-324.

Richardson, J. (2005). Instruments for obtaining student feedback: a review of the literature. Assessment y Evaluation in Higher Education, 30(4), 387-415.

Ricketts, Ch., y Zakrzewski, S. (2005). A risk-analysis approach to implementing web-based assessment. Assessment y Evaluation in Higher Education, 30(6), 603-620.

Romer, T. (2002). Situated learning and assessment. Assessment y Evaluation in Higher Education, 27(3), 233-241.

Rust, C., O'Donovan, B., y Price, M. (2005). A social constructivist assessment process model: how the research literature shows us this could be best practice. Assessment $y$ Evaluation in Higher Education, 30(3), 231-240.

Sambell, K. y McDowell, L (1998). The construction of the hidden curriculum: messages and meanings in the assessment of student learning. Assessment y Evaluation in Higher Education, 23(4), 391-402.

Sander, P. (2005). La investigación sobre nuestros alumnos, en pro de una mayor eficacia en la enseñanza universitaria, Electronic Journal of Research in Educational Psychology, 3(1), 113-130.

Santos Guerra, M. A. (2003). Una flecha en la Diana. La evaluación como aprendizaje. Madrid: Narcea.

Schelfhout, W., Dochy, F., y Janssens, S. (2004). The use of self, peer and teacher assessment as a feedback system in a learning environment aimed at fostering skills of cooperation in an entrepreneurial context. Assessment y Evaluation in Higher Education, 29(2), 177-201.

Segers, M., y Dochy, F. (1996). Quality assurance in higher education: theoretical considerations and empirical evidence. Studies in Educational Evaluation, 22 (2), 115-137.

Sheard, J., y Markham, S. (2005). Web-based learning environments: developing a framework for evaluation. Assessment y Evaluation in Higher Education, 30(4), 353-368.

Silén, Ch. (2006). The Tutor's Approach in Base Groups (PBL). Higher Education, 51(3), 373-385.

Smith, K., y Tillema, H. (2003). Clarifying different types of portfolio use. Assessment y Evaluation in Higher Education, 28(6), 625-648.

Smyth, K. (2004). The benefits of students learning about critical evaluation rather than being summatively judged. Assessment y Evaluation in Higher Education, 29(3), 370-378. 
Somervell, H. (1993). Issues in Assessment, Enterprise and Higher Education: the case for self-peer and collaborative assessment. Assessment y Evaluation in Higher Education, $18(3), 221-233$.

Starr-Glass, D. (2005). Metaphors and maps in evaluation. Assessment y Evaluation in Higher Education, 30(2), 195-207.

Stefani, L. (1998). Assessment in partnership with learners. Assessment y Evaluation in Higher Education, 23(4), 339-350.

Stone, J., y Friedman, S. (2002). A Case Study in the Integration of Assessment and General Education: lessons learned from a complex process. Assessment y Evaluation in Higher Education, 27(2), 199-211.

Stowell, M. (2004).Equity, justice and standards: assessment decision making in higher education. Assessment y Evaluation in Higher Education, 29(4), 495-510.

Struyven, K., Dochy, K., y Janssens, S. (2005). Students' perceptions about evaluation and assessment in higher education: a review. Assessment y Evaluation in Higher Education, 30(4), 325-341.

Sullivan, K. (2002). Credit and Grade Transfer within the European Union's SOCRATES Programme: unity in diversity or head in the sand?. Assessment y Evaluation in Higher Education, 27(1), 65-74.

Sumsion, J., y Fleet, A. (1996). Reflection: can we assess it? Should we assess it?. Assessment y Evaluation in Higher Education, 21(2), 121-130.

Taras, M. (2001). The Use of tutor feedback and student self-assessment in summative assessment tasks: towards transparency for students and for tutors. Assessment y Evaluation in Higher Education, 26(6), 605-614.

Taras, M. (2002). Using assessment for learning and learning from assessment. Assessment y Evaluation in Higher Education, 27(6), 501-510.

Taras, M. (2003). To feedback or not to feedback in student self-assessment. Assessment $y$ Evaluation in Higher Education, 28(5), 549-565.

Taylor, K., y Marienau, C. (1997). Constructive-development theory as a framework for assessment in Higher Education. Assessment y Evaluation in Higher Education, 22(2), 233-243.

Thomson, K., y Falchikov, N. (1998) "Full on until the sun comes out": the effects of assessment on student approaches to studying. Assessment y Evaluation in Higher Education, 23(4), 379-390. 
Tian, X. (2007). Do assessment methods matter? A sensitivity test. Assessment y Evaluation in Higher Education, 32(4), 387-401.

Toohey, S. (2002). Assessment of students' personal development as part of preparation for professional work--is it desirable and is it feasible?. Assessment $y$ Evaluation in Higher Education, 27(6), 529-538.

Topping, K. (1998). Peer assessment between students in colleges and universities. Review of Educational Research, 68 (3), 249-276.

Torrano, F. y González, M. (2004). El aprendizaje autorregulado: presente y futuro de la investigación, Electronic Journal of Research in Educational Psychology, 2(1), 1-34.

Tractenberg, R., Chaterji, R., y Haramati, A. (2007). Assessing and analyzing change in attitudes in the classroom. Assessment y Evaluation in Higher Education, 32(2), 107-120.

Trotter, E. (2006). Student perceptions of continuous summative assessment. Assessment y Evaluation in Higher Education, 31(5), 505-521.

Van Eekelen, I., Boshuizen, H. y Vermunt, J. (2005). Self-regulation in Higher Education Teacher Learning. Higher Education, 50(3), 447-471.

Wagner, Z. (1999). Using student journals for course evaluation. Assessment y Evaluation in Higher Education 24(3), 261-272.

Walden, K., y Peacock, A. (2006). The i-Map: a process-centered response to plagiarism. Assessment y Evaluation in Higher Education, 31(2), 201-214.

Walsh, A. (2007). An exploration of Biggs' constructive alignment in the context of workbased learning. Assessment y Evaluation in Higher Education, 32(1), 79-87.

Watt, S., Simpson, C., McKillop, Ch., y Nunn, V. (2002). Electronic Course Surveys: does automating feedback and reporting give better results?. Assessment y Evaluation in Higher Education, 27(4), 325-337.

Weaver, M. (2006). Do students value feedback? Student perceptions of tutors' written responses. Assessment y Evaluation in Higher Education, 31(3), 379-394.

Weber, L., Frary, R.; Cross, L. (1995). Allowing students a choice of items on objective examinations. Assessment y Evaluation in Higher Education, 20 (3), 301-306.

Webster, F., Pepper, D., y Jenkins, A. (2000). Assessing the undergraduate dissertation. Assessment y Evaluation in Higher Education, 25(1), 1-80.

Welsh, J. (2002). Assessing the transfer function: benchmarking best practices from state Higher Education agencies. Assessment y Evaluation in Higher Education, 27(3), $257-$ 268 . 
Welsh, J., Alexander, S., y Dey, S. (2001). Continuous Quality Measurement: restructuring assessment for a new technological and organisational environment. Assessment y Evaluation in Higher Education, 26(5), 391-401.

Williams, J. (2006). Assertion-reason multiple-choice testing as a tool for deep learning: a qualitative analysis. Assessment y Evaluation in Higher Education, 31(3), 287-301.

Wilson, K., y Fowler, J. (2005). Assessing the impact of learning environments on students' approaches to learning: comparing conventional and action learning designs. Assessment y Evaluation in Higher Education, 30(1), 87-101.

Wilson, M y Scalise, K. (2006). Assessment to improve learning in higher education: The BEAR Assessment System. Higher Education, 52(4), 635-653.

Winning, T., Lim, E., y Townsend, G. (2005). Student experiences of assessment in two problem-based dental curricula: Adelaide and Dublin. Assessment y Evaluation in Higher Education, 30 (5), 489-505.

Winter, R. (1994). Work-based Learning and Quality Assurance in Higher Education. Assessment y Evaluation in Higher Education, 19(3), 247-257.

Woodward, H. (1998). Reflective Journals and Portfolios: learning through assessment. Assessment y Evaluation in Higher Education, 23(4), 415-423.

Woodward, H. y Nanlohy, Ph. (2004). Digital portfolios: fact or fashion?. Assessment y Evaluation in Higher Education, 29(2), 227-238.

Woolf, H. (2004). Assessment criteria: reflections on current practices. Assessment y Evaluation in Higher Education, 29(4), 479-493.

Yorke, M (1998). The management of assessment in higher education. Assessment and Evaluation in Higher Education, 23(2), 101-16.

Yorke, M. (2003). Formative assessment in higher education: Moves towards theory and the enhancement of pedagogic practice. Higher Education, 45(4), 477-501.

Zakrzewski, S., y Bull, J. (1998). The mass implementation and evaluation of computerbased assessments. Assessment y Evaluation in Higher Education, 23(2), 141-152. 
Ibis Álvarez

[Página en blanco por razones de paginación] 